\title{
A sparse stiff chemistry solver based on dynamic adaptive integration for efficient combustion simulations
}

\author{
Chao Xu' $u^{\mathrm{a}}$, Yang Gao ${ }^{\mathrm{a}}$, Zhuyin Ren ${ }^{\mathrm{b}}$, Tianfeng $\mathrm{Lu}^{\mathrm{a}}{ }^{\text {* }}$ \\ a) Department of Mechanical Engineering, University of Connecticut, Storrs, CT 06269- \\ 3139, USA \\ b) Center for Combustion Energy and School of Aerospace Engineering, Tsinghua \\ University, Beijing 100084, China
}

Submitted to Combustion and Flame

Article type: Full length

*Corresponding author:

E-mail: tlu@engr.uconn.edu

Phone: (860) 486-3942

Fax: (860) 486-5088 


\begin{abstract}
A sparse stiff chemistry solver based on dynamic adaptive hybrid integration (AHI-S) is developed and demonstrated for efficient combustion simulations. In a previous study, a dynamic adaptive method for hybrid integration (AHI) was developed to speed up the time integration of chemically reacting flows with detailed chemistry. The AHI method solves the fast subcomponent of chemistry implicitly and the slow subcomponent of chemistry and transport explicitly, and it was shown that AHI is more accurate and efficient than the operator-splitting schemes when there are significant radical sources from the transport term. In the present study, the AHI method is first improved to minimize the number of nontrivial entries in the Jacobian. Sparse matrix techniques are further integrated into AHI to achieve high computational efficiency. The performance of the new AHI-S solver is investigated in constant-pressure autoignition systems using different mechanisms that consist of 9 to 2878 species. It is shown that the computational cost of the AHI-S solver is overall linearly proportional to the mechanism size and is comparable to that of evaluating reaction rates using CHEMKIN-II subroutines. The AHI-S solver achieves speed-up factors ranging from approximately 10, for the 9-species hydrogen mechanism, to approximately 3000, for the 2878-species biodiesel mechanism, compared with the fully implicit VODE solver with Jacobian evaluated through numerical perturbations and factorized with dense matrix operations. It is further found that for mechanisms with less than approximately 100 species, the time saving of AHI-S is primarily attributed to the reduced size of the implicit core of the governing equations, while for mechanisms with more than 100 species, the computational cost of VODE is dominated by the dense LU factorization, such that the time saving of AHI-S is mostly attributed to the sparse LU factorization. The AHI-S solver is then applied to unsteady perfectly stirred reactors involving extinction and re-ignition. Speed-up
\end{abstract}


factors from 50 to 30,000 are achieved compared with the Strang splitting scheme with the chemistry substeps implicitly integrated with VODE, while speed-up factors of 10 100 are achieved compared with the Strang splitting scheme implemented with the sparse stiff LSODES solver. In the end, the performance of AHI-S is investigated in one-dimensional (1-D) unsteady freely propagating laminar premixed flames for a methane/air mixture, for which the time step size in AHI-S is limited by the fastest transport process. A speed-up factor of approximately 200 is achieved compared with the Strang splitting scheme for fixed time step sizes between $10^{-8} \mathrm{~S}$ and $10^{-6} \mathrm{~s}$.

Keywords: sparse ODE solver, dynamic adaptive hybrid integration, stiff chemistry, detailed mechanisms, chemically reacting flow 


\section{Introduction}

Detailed chemical kinetics is important for accurate prediction of combustion processes such as ignition, extinction and flame propagation, while it may involve a large number of species and reactions [1]. Recent progress in mechanism reduction, e.g. using directed relation graph (DRG) based methods [2-7], makes it possible to obtain accurate yet still comprehensive skeletal mechanisms with less than a few hundreds of species for practical engine fuels. However chemical stiffness may remain in skeletal and even reduced mechanisms due to the highly reactive radicals and their short timescales, such that the high-cost implicit solvers for ordinary differential equations (ODE), e.g. VODE [8] and DASAC [9], are typically required for time integration of combustion systems using reasonably large time steps.

To alleviate this problem, dynamic stiffness removal [10] was developed to eliminate short chemical timescales for compressible flow simulations using direct numerical simulations (DNS), such that the low-cost explicit solvers can be employed with time step sizes up to approximately 20 ns. Implicit solvers are typically required for combustion simulations involving even larger time steps. For multidimensional flows, the operator-splitting schemes are widely used to separate chemistry integration from that of transport processes to avoid the high computational cost for solving fully coupled implicit equations [11-15]. Splitting schemes however can incur significant errors in certain cases. For example, it was found in Ref. [16] that, when significant radical sources are present in the transport term, $O(1)$ splitting errors may occur in ignition processes unless small time steps comparable to those required for explicit solvers are taken. A dynamic adaptive hybrid integration (AHI) method [16] was then developed for effective error

control in such cases. Significant speedup was achieved as well using AHI compared with the 
splitting schemes for small and moderately large mechanisms, say with less than about 100 species, by solving only the fast sub-component of chemistry implicitly.

Nevertheless, for large mechanisms, Jacobian evaluation and factorization can dominate the computational cost in implicit solvers, such that analytical Jacobian evaluation [17], sparse matrix techniques [18-20], and adaptive preconditioning methods [21, 22] can significantly speed up stiff chemistry solvers. As reported in Refs. [18, 20], the computational cost for chemistry integration can be reduced to approximately a linear function of the number of species, $N$, using sparse matrix techniques, while it can scale as $O\left(N^{2}\right)$ to $O\left(N^{3}\right)$ using dense matrix operations. The sparse matrix techniques are also applicable to AHI to further reduce the computational cost, particularly when large mechanisms are involved.

In the present study, a sparse AHI solver (AHI-S) is developed to further reduce the size of the fast chemistry subcomponent, such that sparser Jacobian can be obtained for improved efficiency. The performance of the AHI-S solver is first investigated in auto-ignition using mechanisms with 9, for hydrogen, to 2878 species, for a biodiesel surrogate. AHI-S is compared with other solvers for numerical efficiency in a variety of combustion systems including autoignition, unsteady perfectly stirred reactors (PSRs) and 1-D freely propagating premixed flames. It will be shown that AHI-S can achieve similar per-step computational cost to that of fully explicit solvers.

The paper is organized as follows. In Section 2, the AHI method is reviewed and the AHI-S method is formulated, results from the AHI-S method for various combustion systems are presented and compared with other solvers in Section 3, and conclusions are drawn in Section 4.

\section{Methodology}




\subsection{Review of the AHI method}

The spatially discretized governing equations for typical reacting flows can be expressed as the following ODEs:

$$
\frac{d \boldsymbol{\Phi}}{d t}=\boldsymbol{S}(\boldsymbol{\Phi})+\boldsymbol{M}(\boldsymbol{\Phi})
$$

where $\boldsymbol{\Phi}$ is the vector of dependent variables of dimension $n_{\Phi}$, including, e.g. temperature and species concentrations, and $\boldsymbol{S}$ and $\boldsymbol{M}$ represent the chemistry and transport terms, respectively. Note that in a multi-grid system, $\boldsymbol{\Phi}$ consists of variables at all grid points. In the AHI method [16], fast species and reactions are first identified on-the-fly based on reaction timescales defined in a recent analytic formulation of computational singular perturbation (CSP) [23]:

$$
\begin{gathered}
\tau_{i} \equiv\left|\frac{\partial \Omega_{i}}{\partial \boldsymbol{c}} \cdot \boldsymbol{v}_{i}\right|^{-1}, \\
\frac{\partial \Omega_{i}}{\partial \boldsymbol{c}}=\left[\frac{\partial \Omega_{i}}{\partial c_{1}}, \frac{\partial \Omega_{i}}{\partial c_{2}}, \ldots, \frac{\partial \Omega_{i}}{\partial c_{N}}\right], \quad \boldsymbol{v}_{i}=\left[v_{1, i}, v_{2, i}, \ldots, v_{N, i}\right]^{T}
\end{gathered}
$$

where $\tau_{i}$ is the timescale of the $i$ th reaction, $\Omega_{i}$ is the reaction rate, $N$ is the number of species, $c_{k}$ is the mole concentration of the $k$ th species, and $v_{k, i}$ is the stoichiometric coefficient of the $k$ th species in the ith reaction. The $i$ th reaction is considered to be fast if

$$
\tau_{i}<\tau_{c} / \beta
$$

where $\tau_{c}$ is a threshold timescale that is typically comparable to the integration time step $\Delta t$, and $\beta$ is a safety factor. The $k$ th species is considered to be fast if the following criterion is satisfied for any fast reaction $i$,

$$
\left|\frac{\partial \Omega_{i}}{\partial c_{k}}\right|>\beta \tau_{c}^{-1}
$$

Note that the safety factor is typically mechanism-dependent and different safety factors can be optionally used for the identification of fast species and reactions. 
The governing equations (Eq. 1) can then be rewritten as:

$$
\begin{gathered}
\frac{d \boldsymbol{\Phi}}{d t}=\boldsymbol{S}_{f}(\boldsymbol{\Phi})+\mathbf{g}_{s}(\boldsymbol{\Phi}), \quad \boldsymbol{\Phi}=\left[\begin{array}{l}
\boldsymbol{\Phi}_{f} \\
\boldsymbol{\Phi}_{s}
\end{array}\right] \\
\boldsymbol{S}_{f}=\sum_{i=1}^{m} \boldsymbol{v}_{i} \Omega_{i} \\
\mathbf{g}_{s}=\sum_{i=m+1}^{n_{r}} \boldsymbol{v}_{i} \Omega_{i}+\boldsymbol{M}
\end{gathered}
$$

where $\boldsymbol{\Phi}_{f}$ and $\boldsymbol{\Phi}_{s}$ are the fast and slow variables of dimension $n_{f}$ and $n_{\Phi}-n_{f}$, respectively, $n_{r}$ is the total number of reactions, and $m$ is the number of fast reactions. It is assumed that sufficiently small integration time steps are taken, such that the transport term and the energy equation are not stiff. In AHI, the ODE system in Eq. (5) is temporally discretized using a firstorder scheme:

$$
\left[\begin{array}{l}
\boldsymbol{\Phi}_{f}^{n+1}-\boldsymbol{\Phi}_{f}^{n} \\
\boldsymbol{\Phi}_{s}^{n+1}-\boldsymbol{\Phi}_{s}^{n}
\end{array}\right] \frac{1}{\Delta t}=\mathbf{S}_{f}\left(\boldsymbol{\Phi}_{f}^{n+1}, \boldsymbol{\Phi}_{s}^{n}\right)+\boldsymbol{g}_{s}\left(\boldsymbol{\Phi}_{f}^{n}, \boldsymbol{\Phi}_{s}^{n}\right)
$$

where the superscript $n$ indicates the $n$th time step. The fast variables $\boldsymbol{\Phi}_{f}^{n+1}$ are solved implicitly using the first $n_{f}$ equations of Eq. (6). The slow variables can then be solved explicitly from the remaining equations.

\subsection{A sparse AHI solver (AHI-S)}

\subsubsection{Sparse Jacobian for the fast chemistry subcomponent}

The chemical Jacobian is typically sparse for most practical fuels involving large mechanisms. The sparse pattern of the chemical Jacobian is first demonstrated using constantpressure auto-ignition governed by 


$$
\begin{gathered}
\frac{d y_{k}}{d t}=\frac{\omega_{k} W_{k}}{\rho}, k=1,2, \ldots, N \\
\frac{d T}{d t}=-\frac{1}{\rho c_{p}} \sum_{k=1}^{N} h_{k} \omega_{k} W_{k} \\
\rho=p /\left(R_{u} T \sum_{k=1}^{N} \frac{y_{k}}{W_{k}}\right)
\end{gathered}
$$

where the subscript $k$ indicates the $k$ th species, $y$ is mass fraction, $\omega$ is species molar production rate, $W$ is molecular weight, $h$ is specific enthalpy, $c_{p}$ is the mixture-averaged specific heat capacity, $\rho$ is density, $p$ is pressure, $T$ is temperature, and $R_{u}$ is the universal gas constant.

The Jacobian for Eqs. (7-8) is typically dense if the dependent variable vector $\boldsymbol{\Phi}$ consists of only species mass fractions, $y_{k}$, and temperature, $T$, because all the variables are coupled through Eq. (9). As a solution provided in Ref. [19], sparse chemical Jacobian can be obtained by including density as a dependent variable and replacing Eq. (9) with the following differential equation:

$$
\frac{d \rho}{d t}=\frac{1}{c_{p} T} \sum_{k=1}^{N} h_{k} \omega_{k} W_{k}-\left(\sum_{k=1}^{N} \frac{y_{k}}{W_{k}}\right)^{-1} \sum_{k=1}^{N} \omega_{k}
$$

Similarly, since species can be coupled through third bodies in pressure-dependent reactions, including the total third body concentration as a dependent variable can further reduce the number of nontrivial entries in the Jacobian [19]. More specifically, the equation of state,

$$
C=p / R_{u} T
$$

where $C$ is the total mole concentration, can be replaced with the following differential equation

$$
\frac{d C}{d t}=\frac{C}{\rho c_{p} T} \sum_{k=1}^{N} h_{k} \omega_{k} W_{k}
$$


The ODE system including Eqs. $(7-8,10,12)$ can then be solved for the set of dependent variables $\boldsymbol{\Phi}=\left[y_{k}, T, \rho, C\right]^{\mathrm{T}}$. For simplicity, density and total mole concentration are treated as slow variables in the present study by taking sufficiently small integration time steps. Note that either Eqs. $(9,11)$ or Eqs. $(10,12)$ can be used for updating slow variables $\rho$ and $C$. Since only the fast chemistry subcomponent is implicitly solved, AHI only needs to evaluate and factorize the Jacobian for the fast chemistry subcomponent, which is typically still sparse when Eqs. (10, 12) are solved together with Eqs. (7-8). As such, high computational efficiency can be achieved by combining sparse Jacobian techniques with AHI.

\subsubsection{Minimizing the size of the fast chemistry subcomponent}

To achieve an optimal efficiency of AHI-S, the number of nontrivial entries in the Jacobian for the fast chemistry subcomponent in Eqs. $(7-8,10,12)$ is further minimized in AHI-S based on the observation that, the rate of each elementary reaction is typically sensitive to concentrations of only one or two fast species, resulting in few large entries in the Jacobian, while the weak dependency of the reactions on slow species concentrations results in relatively small entries with insignificant contribution to the chemical stiffness. When multiple reactions are involved, a species can be fast for one reaction but slow for the others, based on the definition in Eq. (4). Compared with the original AHI method, in which the Jacobian of the fast chemistry subcomponent is dense and may consist of both large and small entries, AHI-S systematically eliminates the unimportant entries from the Jacobian to achieve a higher computational efficiency.

Procedurally, the fast reactions are first identified with Eq. (3) in AHI-S to separate the fast and slow source terms. The fast species are then identified with Eq. (4) to separate the fast 
variables $\boldsymbol{\Phi}_{f}$ and slow variables $\boldsymbol{\Phi}_{\mathrm{s}}$ to obtain Eq. (5). Note that this procedure is same as that in AHI. A set of reaction-specific fast species, $\boldsymbol{\Phi}_{f}^{\langle i\rangle}$ as a subset of all the fast variables $\boldsymbol{\Phi}_{f}$ is then identified for the ith fast reaction based on their contribution to the reaction timescale. That is, the $k$ th species is included in $\boldsymbol{\Phi}_{f}^{\langle i\rangle}$ if and only if Eq. (4) is satisfied for the ith fast reaction. The remaining species are then defined as reaction-specific slow species, $\boldsymbol{\Phi}_{s}^{\langle i\rangle}$. Equation (5) is then temporally discretized as

$$
\begin{gathered}
{\left[\begin{array}{l}
\boldsymbol{\Phi}_{f}^{n+1}-\boldsymbol{\Phi}_{f}^{n} \\
\boldsymbol{\Phi}_{s}^{n+1}-\boldsymbol{\Phi}_{s}^{n}
\end{array}\right] \frac{1}{\Delta t}=\mathbf{S}_{f}+\boldsymbol{g}_{s}} \\
\boldsymbol{S}_{f}=\sum_{i=1}^{m} \boldsymbol{v}_{i} \Omega_{i}\left(\boldsymbol{\Phi}_{f}^{\langle i\rangle, n+1}, \boldsymbol{\Phi}_{s}^{\langle i\rangle, n}\right) \\
\mathbf{g}_{s}=\sum_{i=m+1}^{n_{r}} \boldsymbol{v}_{i} \Omega_{i}\left(\boldsymbol{\Phi}_{f}^{n}, \boldsymbol{\Phi}_{s}^{n}\right)+\boldsymbol{M}\left(\boldsymbol{\Phi}_{f}^{n}, \boldsymbol{\Phi}_{s}^{n}\right)
\end{gathered}
$$

where the superscript $n$ indicates the $n$th time step, the superscript $\langle i\rangle$ indicates the $i$ th fast reaction, $\boldsymbol{\Phi}_{f}^{\langle i\rangle}, \boldsymbol{\Phi}_{s}^{\langle i\rangle}$ are reaction-specific fast and slow variables of dimension $n_{f}^{\langle i\rangle}$ and $n_{\Phi}-n_{f}^{\langle i\rangle}$, respectively. Based on Eq. (13), a fast species is not treated implicitly for every fast reaction. Instead, only the contribution from reaction specific fast species are included in the Jacobian evaluation for a specific fast reaction, such that the Jacobian of fast chemistry using Eq. (13) can be significantly sparser than that of Eq. (6) used in the original AHI formulation. 
a)

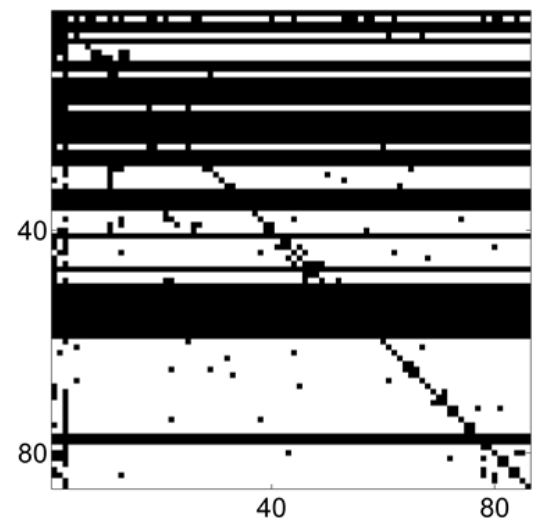

b)

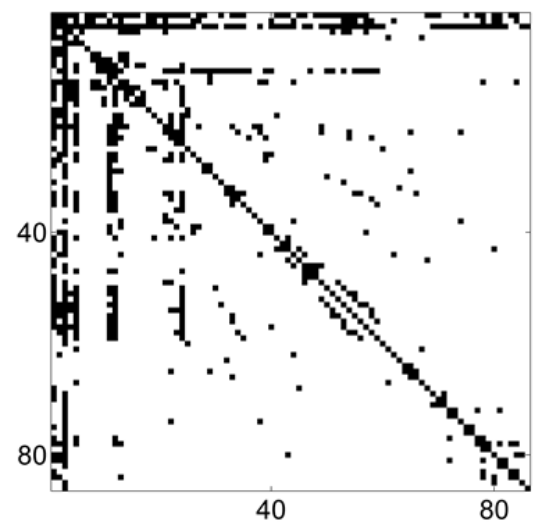

c)

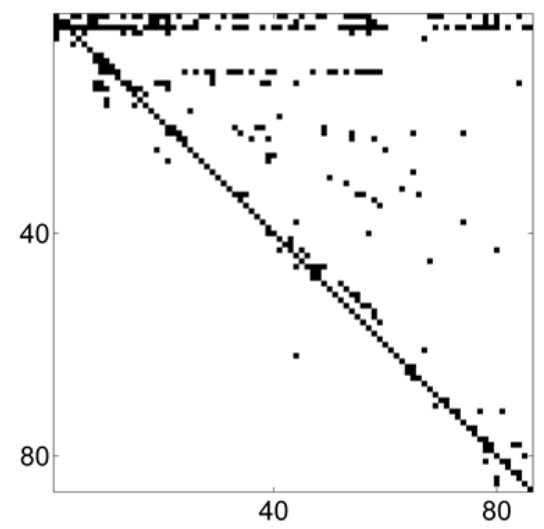

Figure 1. Patterns of the nontrivial entries (black pixels) in the Jacobian of fast chemistry for Eqs. (7-8) evaluated a) with Eq. (10) but without Eqs. (12-13), b) with Eqs. $(10,12)$ but without Eq. (13), and c) with Eqs. $(10,12-13)$. The Jacobian is calculated at $t=2 \tau_{\text {ign }}$, where $\tau_{\text {ign }}$ is the ignition delay, for methane/air auto-ignition at constant pressure of $50 \mathrm{~atm}$, equivalence ratio of 0.5 and initial temperature of $1200 \mathrm{~K}$. The integration time step size is $\Delta t=10^{-7} \mathrm{~s}$. The $x$ and $y$ axes represent the indices of the 86 fast species.

To demonstrate the effectiveness of the above strategy to obtain sparse Jacobian, Fig. 1 shows the patterns of nontrivial entries in the Jacobian of fast chemistry with three different formulations in Eqs. (7-8, 10), Eqs. (7-8, 10, 12) and Eqs. (7-8, 10, 12-13), respectively, for constant-pressure methane/air auto-ignition simulated with USC-Mech II [24]. It is seen that adding density and the third body concentration as new dependent variables results in a significantly sparser Jacobian and using Eq. (13) can further reduce the number of nontrivial entries compared with the original AHI formulation in Eq. (6). Sparse linear algebra subroutines, such as that implemented in the Harwell MA48 libraries [25], can be exploited to take advantage of the sparse Jacobian for improved computational efficiency. In the present study, mechanismspecific sparse linear algebra subroutines are systematically generated using an in-house code to 
maximize the efficiency for solving the implicit core for the fast chemistry subcomponent, and the mechanism-specific sparse solver can be readily implemented for different reacting flows, such as unsteady PSRs and 1-D premixed flames as demonstrated in the following.

\section{Results and Discussion}

The newly developed AHI-S solver is tested with eight different mechanisms listed in Table 1 in different combustion systems including constant-pressure auto-ignition, unsteady PSRs and 1-D premixed flames. The number of species ranges from 9 for hydrogen to 2878 for a biodiesel surrogate. Flame conditions involved, including pressure $(p)$, initial/inlet temperature $\left(T_{0}\right)$ in auto-ignition/unsteady PSRs, equivalence ratio $(\phi)$ and safety factors $(\beta)$, are also shown in Table 1. Based on all the mechanisms we studied, $\beta=0.4 \sim 0.5$ is recommended for mechanisms with fewer than about 100 species, while for larger mechanisms, $\beta=0.1 \sim 0.2$ is found to be safe. A fixed time step size of $10^{-7} \mathrm{~s}$ is adopted for all the calculations unless otherwise specified. The simulation time for auto-ignition is five times the ignition delay $\left(\tau_{i g n}\right)$, while the simulation time for unsteady PSRs is twice the fluctuation period of residence time, which will be further explained in Section 3.2. All the numerical codes are implemented in FORTRAN and compiled with the Intel FORTRAN Compiler and tested on Intel CPUs.

Table 1: Mechanisms and parameters tested in auto-ignition and unsteady PSRs 


\begin{tabular}{cccccc}
\hline Mechanisms & $\begin{array}{c}\text { Number of } \\
\text { species }\end{array}$ & $\boldsymbol{p ( a t m )}$ & $\boldsymbol{T}_{\mathbf{0}}(\mathbf{K})$ & $\boldsymbol{\phi}$ & $\boldsymbol{\beta}$ \\
\hline Hydrogen [26] & 9 & 1 & 1200 & 1.0 & 0.5 \\
Ethylene (skeletal) [27] & 32 & 1 & 1200 & 1.0 & 0.5 \\
Methane [24] & 111 & 50 & 1200 & 0.5 & 0.5 \\
iso-Octane (skeletal) [28] & 233 & 10 & 1200 & 0.5 & 0.2 \\
n-Heptane [29] & 540 & 10 & 1200 & 0.5 & 0.2 \\
iso-Octane [30] & 874 & 10 & 1200 & 0.5 & 0.2 \\
n-Tetradecane [31] & 1661 & 10 & 1200 & 0.5 & 0.1 \\
Methyl Decanoate [32] & 2878 & 1 & 1200 & 0.45 & 0.2 \\
\hline
\end{tabular}

\subsection{Performance of AHI-S in auto-ignition}

Due to the similar formulations, the AHI-S solver shares similar accuracy with the original AHI, while the computational efficiency is significantly improved for large mechanisms by taking advantage of the sparse chemical Jacobian. Figure 2 shows the numerical solutions of constant-pressure auto-ignition for ethylene/air (Fig. 2a) and iso-octane/air (Fig. 2b) mixtures, calculated with AHI-S in comparison with the fully implicit VODE solver. The relative and absolute error tolerances for VODE are set to be $10^{-9}$ and $10^{-12}$, respectively, which will also be used in the following simulations using VODE. Close agreement is observed between the solutions obtained with AHI-S and the fully implicit VODE, and this is expected because the utilization of sparse matrix techniques does not compromise the order of accuracy.

Figures $3 \mathrm{a}$ and $3 \mathrm{~b}$ further show the relative errors in ignition delay $\left(\tau_{i g n}\right)$ for different values of integration step size $(\Delta t)$ and safety factor $(\beta)$, for auto-ignition of methane/air calculated using USC-Mech II, where the relative error is defined as

$$
\varepsilon=\frac{\left|\tau_{i g n}-\tau_{i g n}^{0}\right|}{\tau_{i g n}^{0}},
$$

where $\tau_{i g n}^{0}$ is the exact solution. In the fully implicit method, $\beta=0$, because all the species are treated implicitly, and the relative error is shown in Fig. 3a to be linearly proportional to the time 
step size. The relative error in AHI-S nevertheless depends on both time step size and $\beta$, while for a fixed $\beta$ value, the relative error shows approximately linear dependency on time step size, indicating the first-order accuracy. Figure $3 \mathrm{~b}$ further shows that the relative error in AHI-S nonmonotonically depends on the number of fast species, which is in turn determined by time step size and $\beta$. It merits further study to construct high-order AHI solvers, and the following study will be focused on the computational efficiency of the first-order AHI-S.
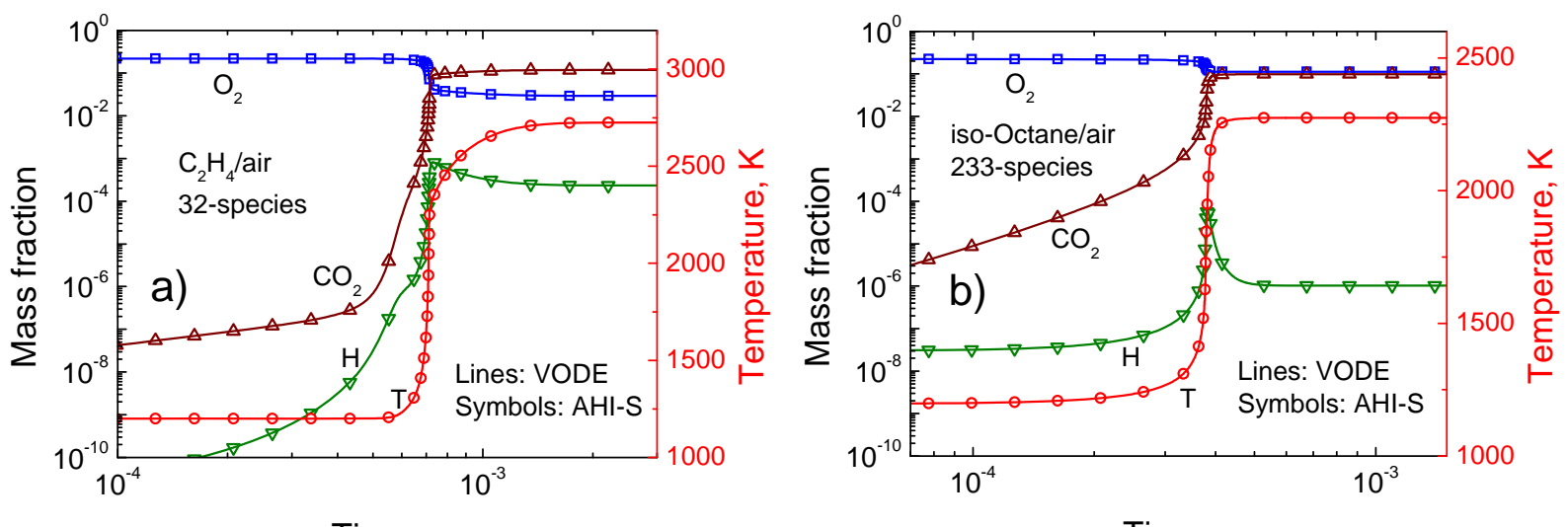

Time, s

Time, s

Figure 2. Species and temperature profiles in constant-pressure auto-ignition for a) a 32-species skeletal mechanism for ethylene and b) a 233-species skeletal mechanism for iso-octane, calculated with the fully implicit VODE solver (solid lines) and the AHI-S solver (symbols), respectively.
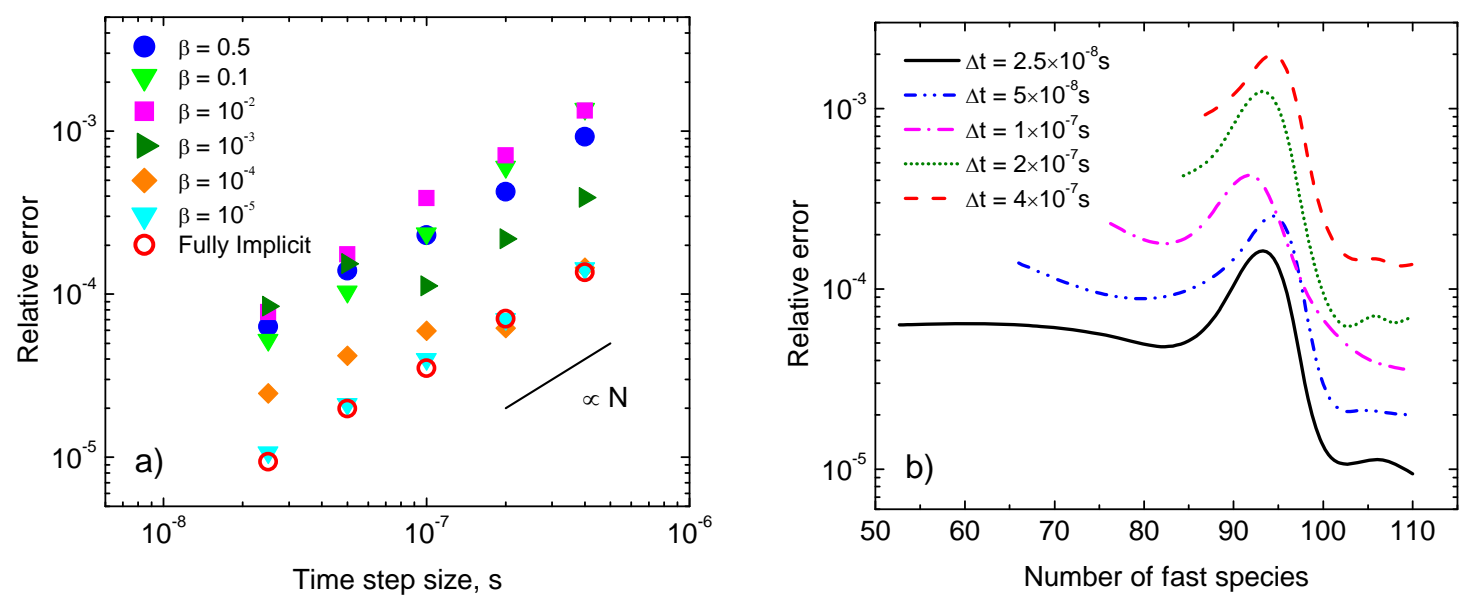
Figure 3. Dependence of relative error in ignition delay time on a) integration step size for different safety factors and b) number of fast species for different integration step sizes, calculated using USC-Mech II at $p=50 \mathrm{~atm}, \phi=0.5, T_{0}=1200 \mathrm{~K}$.

Figure $4 \mathrm{a}$ first shows the numbers of fast species $\left(n_{f}\right)$ and nontrivial entries $\left(n_{n z}\right)$ in the Jacobian of fast chemistry subcomponent in AHI-S as function of mechanism size, averaged over the entire simulation of auto-ignition. Overall linear trends, specifically $n_{f} \approx 0.8 N$ and $n_{n z} \approx$ $3 N$, can be clearly observed for the mechanisms with more than 100 species. Figure $4 \mathrm{~b}$ further shows that the fraction of the nontrivial entries in the Jacobian, $n_{n z} / n_{f}^{2}$, is inversely proportional to the mechanism size, and this trend can be explained by the statistical linear correlation between the number of reactions and number of species observed over a variety of reaction mechanisms and the fact that an elementary reaction involves only a few species on average [1]. Since the computational cost of LU factorization is $O\left(N^{3}\right)$ for dense matrices and can be reduced to $O\left(n_{n z}\right)$ for sparse matrices, speedup factors of $O\left(N^{2}\right)$ can be achieved for large mechanisms by using sparse solvers.
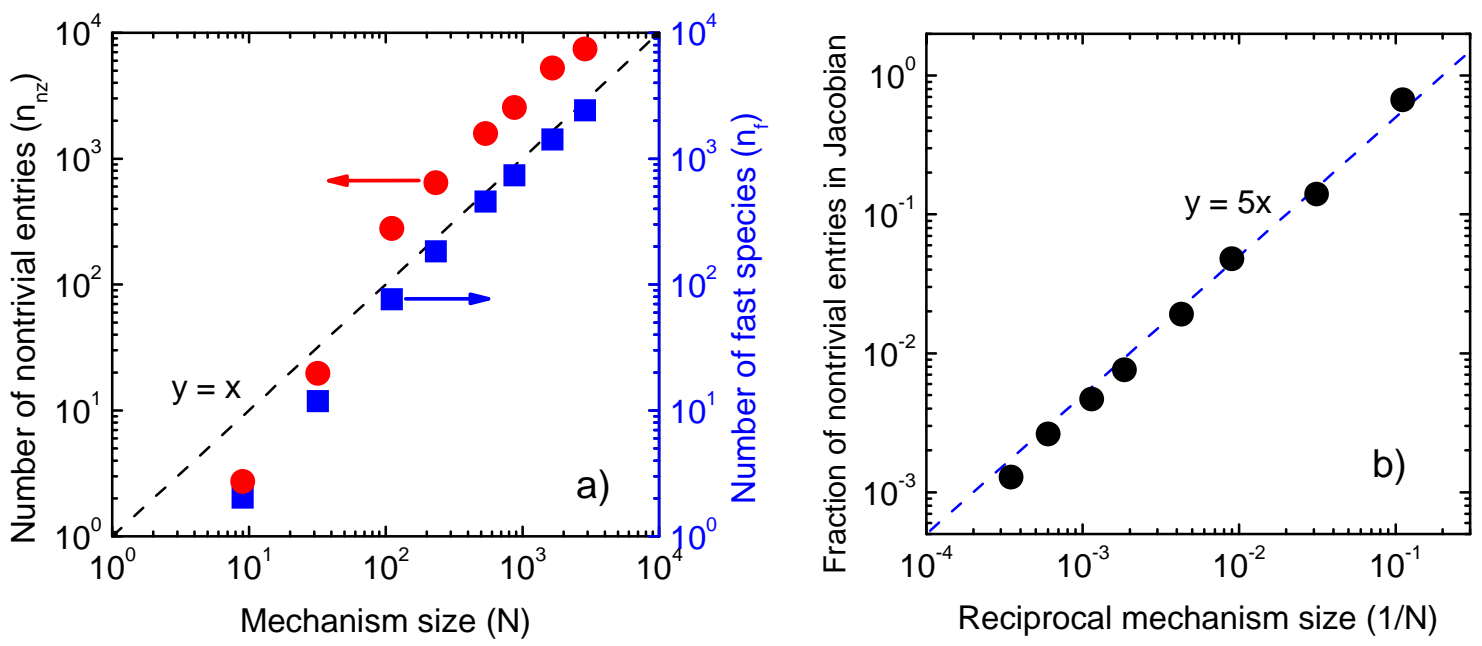
Figure 4. Dependence of a) the numbers of fast species and nonzero entries in the Jacobian in $\mathrm{AHI}-\mathrm{S}$, and b) the fraction of nontrivial entries in the Jacobian, on the total number of species, $N$, for the mechanisms listed in Table 1.

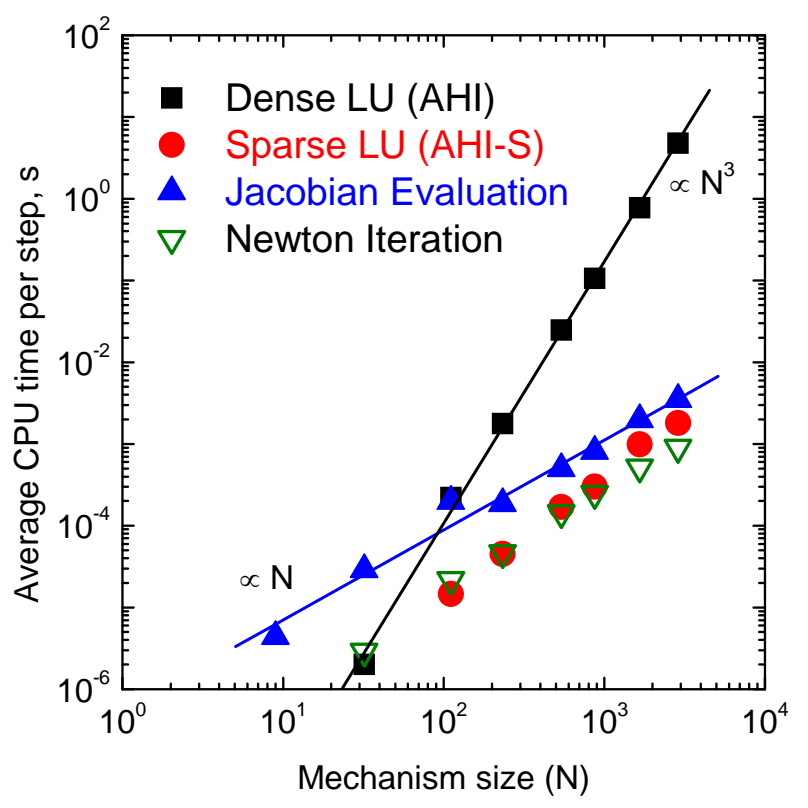

Figure 5. Average per-step computational cost for auto-ignition for the major operations in AHI and AHI-S as function of mechanism size.

Figure 5 shows the computational cost of the major operations in the original AHI and the newly developed AHI-S solver. It is seen that the computational cost of LU factorization is reduced from $O\left(N^{3}\right)$, using dense matrix operations, to $O(N)$ by exploiting sparse matrix techniques. It is further seen that the computational cost of analytic Jacobian evaluation dominates that of sparse LU factorization and Newton's iteration in AHI-S, while in the original AHI method, dense LU factorization is dominant for larger mechanisms, say when $N>100$. 


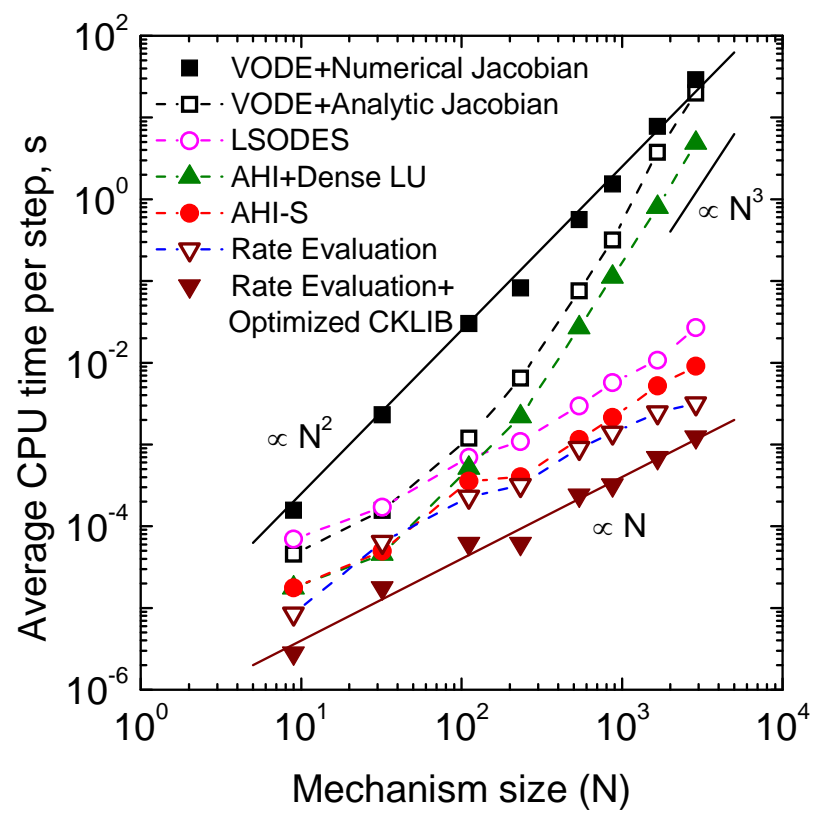

Figure 6. Average per-step computational cost with different ODE solvers as function of mechanism size. A fixed integration time step of $10^{-7} \mathrm{~s}$ is adopted in AHI and AHI-S, and adaptive time stepping is used in VODE and LSODES.

Figure 6 shows the computational cost of different ODE solvers compared with that of evaluating the reaction rates using the CKWYP subroutine in CHEMKIN-II [33] and an optimized in-house rate evaluation subroutine [1], respectively. Among the compared are the fully implicit VODE solvers with numerical and analytic Jacobians, respectively, a fully implicit sparse solver LSODES [34] with analytic Jacobian, the original AHI and the present AHI-S solver. Note that Perini et al. [17] has shown that for 0-D auto-ignition the performance of LSODES is similar to the commercial software CHEMKIN-PRO [35] which appears to use the DASPK solver [36] with a preconditioned Krylov iterative method. The Jacobian reuse feature in VODE and LSODES is switched off for the auto-ignition simulations such that Jacobian evaluation and LU factorization are performed at each integration step. The Jacobian reuse 
feature is unchanged in calculations for unsteady PSRs and 1-D premixed flames when VODE or LSODES is used in the following.

The cost of evaluating the reaction rates approximately indicates the lower limit for the perstep cost of ODE solvers because at least one set of rate evaluations is needed at each time step. Note that while the per-step computational cost of an explicit solver can be comparable to that of one or a few rate evaluations, the implicit solvers can be significantly more expensive due to the additional operations such as Jacobian evaluation and factorization. It is seen in Fig. 6 that the inhouse subroutine for optimized rate evaluation is faster than the CHEMKIN-II subroutine by factors of $2 \sim 5$, and thus similar speedup can be achieved for explicit solvers using the optimized rate subroutine.

In contrast, the fully implicit VODE solver involves time-consuming Jacobian evaluation and LU factorization, and thus the per-step computational cost can be higher than that of explicit solvers by orders of magnitude. Jacobian evaluation through numerical perturbations is typically most time-consuming for small to moderate sized mechanisms, say with less than 100 species, and thus using analytic Jacobian evaluation, which scales as $O(N)$, can significantly reduce the computational cost for such mechanisms. For larger mechanisms, the LU factorization becomes more time-consuming, and thus the speedup factor achieved through analytic Jacobian evaluation decreases with mechanism size and mostly vanishes at about 3000 species. For such large mechanisms, the computational cost for dense LU factorization, which scales as $O\left(N^{3}\right)$, dominates that of both numerical and analytic Jacobian evaluation. In such cases, using sparse matrix techniques can significantly speedup LU factorization and thus the overall integration.

Compared with the full implicit VODE solver using analytic Jacobian, AHI with dense LU factorization can further reduce the computational cost by more than a factor of 2 for 
mechanisms of different sizes, due to the reduced size of the implicit core. It is seen that AHI-S features similar efficiency to that of AHI for small mechanisms, say $N<100$, and can also significantly speedup the integration for large mechanisms, say $N>100$. The computational cost of AHI-S is overall linearly proportional to mechanism size, and is only a few, say about 3 , times that for evaluating the reaction rates using the CHEMKIN II subroutine. While the LSODES solver also features linear computational cost with respect to mechanism size, AHI-S is shown to be faster than LSODES by approximately a factor of three for all the mechanisms as shown in Fig. 6. Therefore, the per-step computational cost of AHI-S is comparable to that of the most efficient fully explicit schemes over the entire range of mechanism size, while much larger integration time steps can be adopted using AHI-S.

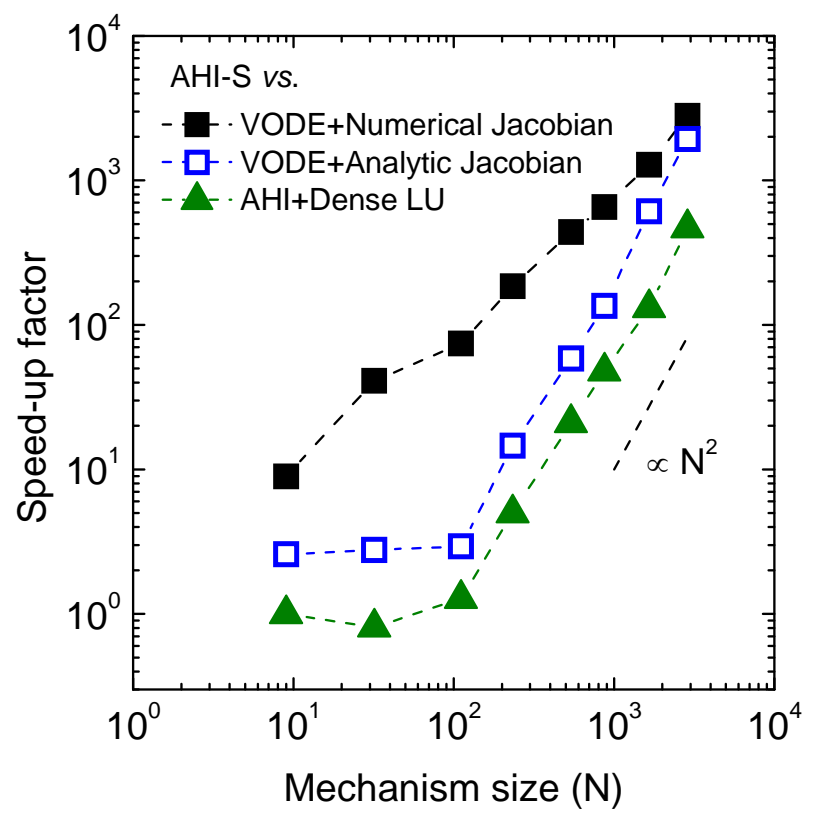

Figure 7. Speed-up factors of AHI-S as function of mechanism size, compared with the original AHI and the fully implicit VODE solver with numerical and analytic Jacobian, respectively. A fixed integration time step of $10^{-7} \mathrm{~s}$ is used for AHI and AHI-S, and the built-in adaptive time stepping algorithm in VODE is used as is. 
Figure 7 further shows the speed-up factors of AHI-S compared with the original AHI and the VODE solver with numerical and analytic Jacobian. It is seen that compared with VODE using numerical Jacobian, AHI-S achieves a speed-up factor of 2800 for the 2878 -species biodiesel mechanism, being even larger than the previously reported speed-up factor of 370 using an adaptive preconditioning method [21]. Compared with the original AHI and VODE with analytic Jacobian, speed-up factors achieved by AHI-S scale as $O\left(N^{2}\right)$ for large mechanisms, reaching about 450 and 2000, respectively, for the biodiesel mechanism.

\subsection{Effect of flame conditions on size of the fast chemistry core}

In AHI-S, the computational efficiency depends on the size of the fast chemistry subcomponent, which can be affected by both the local flame condition and the integration time step size. Figure 8 shows that the average per-step computational cost increases with integration

time step size, pressure, and initial temperature for methane/air auto-ignition, calculated using USC-Mech II. This indicates the sensitivity of the numbers of fast species and reactions to these parameters, as verified in Fig. 9.

To further investigate the effect of local flame condition on the size of the fast chemistry, Fig. 10 shows the patterns of nontrivial entries in the Jacobian before and after ignition for three mechanisms with 32,111 and 233 species, respectively. There are more fast species, as well as nontrivial entries in the Jacobian, in the post-ignition cases than those in the pre-ignition cases for all the three mechanisms. Changes in the pattern of the Jacobian are also observed. It is therefore clear that the speedup achieved by the AHI schemes is sensitive to global and local flame conditions due to the dynamic adaptive nature of the solver. Nevertheless, the Jacobian for 
large mechanisms are almost always sparse, such that significant time savings can almost always be achieved using AHI-S.
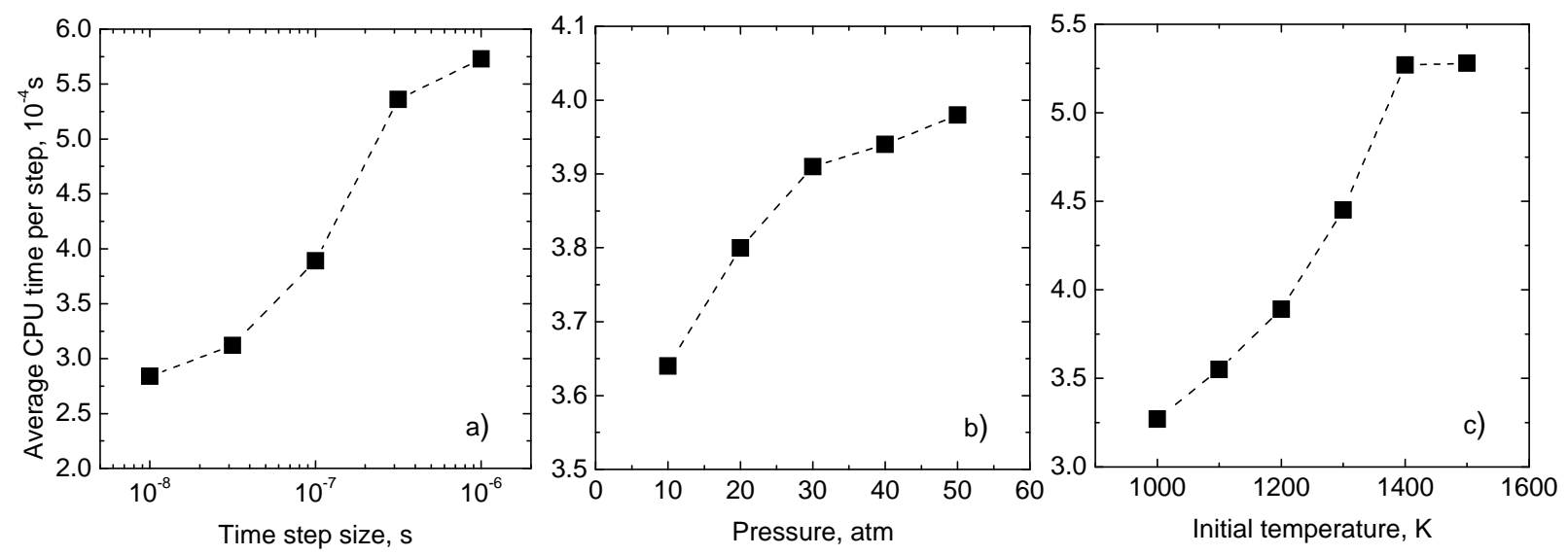

Figure 8. Average per-step computational cost as function of a) time step size, b) pressure, and c) initial temperature, for auto-ignition of methane/air calculated using USC-Mech II. The baseline case is for $p=50 \mathrm{~atm}, \phi=0.5, T_{0}=1200 \mathrm{~K}$, and $\Delta t=10^{-7} \mathrm{~s}$.

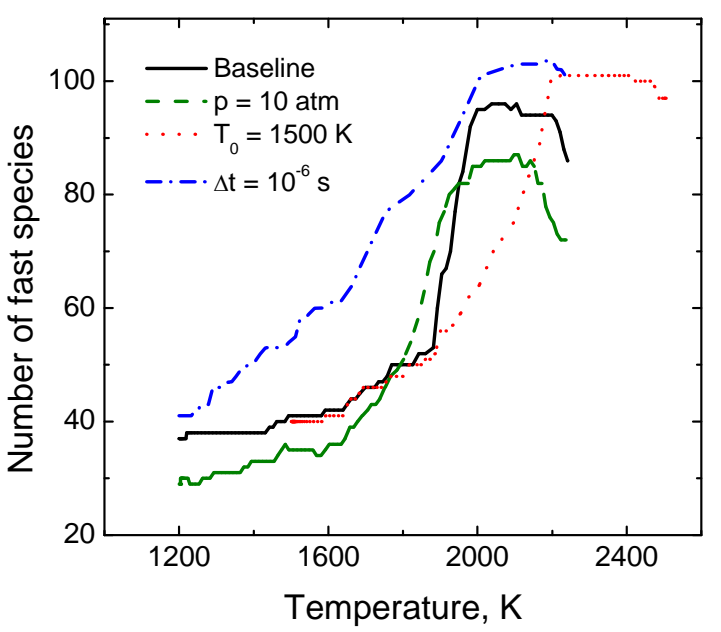

Figure 9. Number of fast species as function of temperature in auto-ignition of methane/air for different time step sizes, pressures and initial temperatures, calculated using USC-Mech II. The baseline case is for $p=50 \mathrm{~atm}, \phi=0.5, T_{0}=1200 \mathrm{~K}$, and $\Delta t=10^{-7} \mathrm{~s}$. 

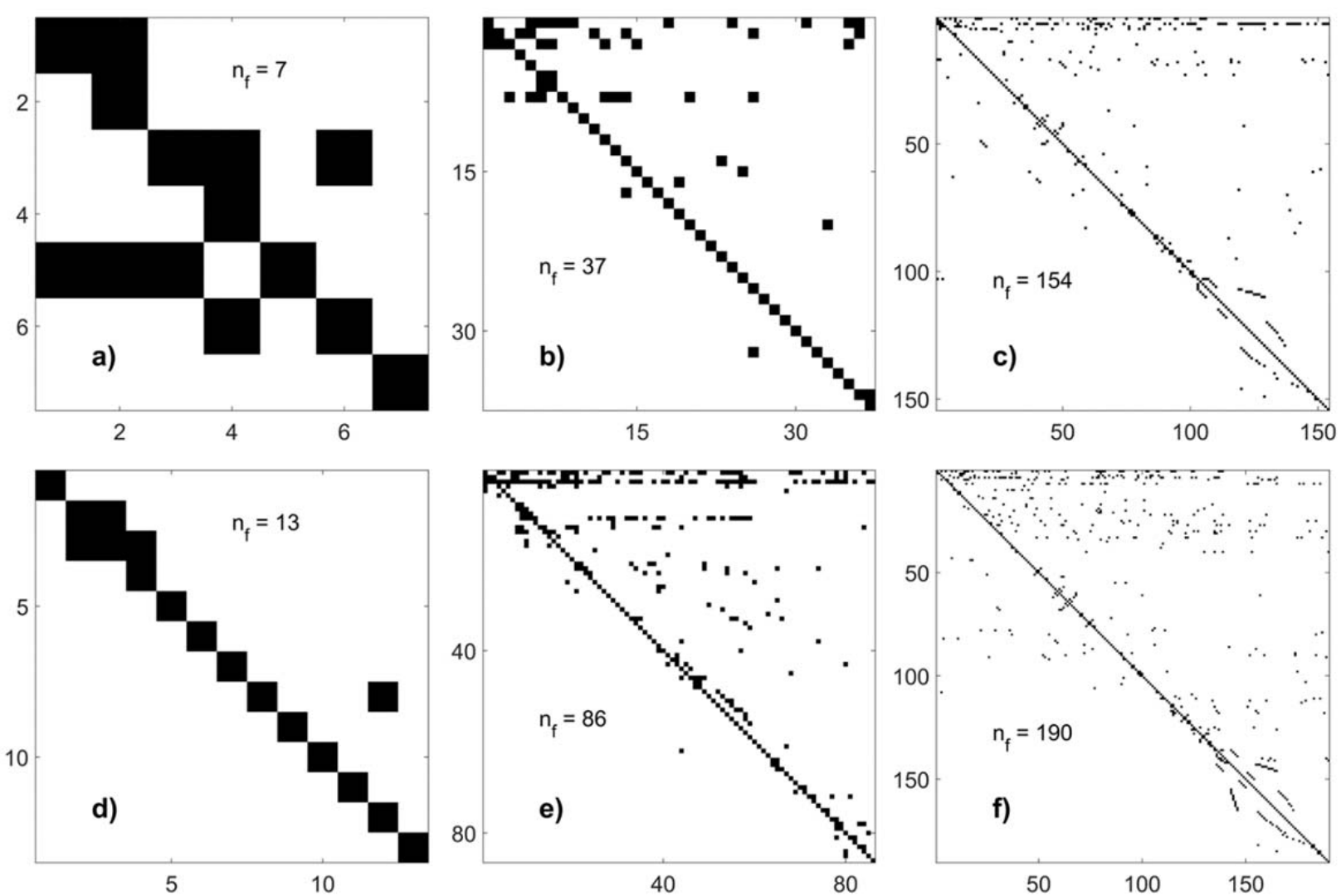

Figure 10. Pattern of nontrivial entries (black pixels) in the Jacobian of the fast chemistry subcomponent for $(\mathrm{a}, \mathrm{d})$ the 32-species skeletal mechanism for ethylene, $(\mathrm{b}, \mathrm{e})$ the 111-species USC-Mech II with methane as fuel, and (c,f) the 233-species skeletal mechanism for iso-octane. The top (a-c) and bottom (d-f) rows are calculated at $t=0.5$ and 2 times the ignition delay, respectively.

\subsection{Performance of AHI-S in unsteady PSRs}

PSR is an important 0-D application that can involve both extinction and ignition due to the presence of homogeneous mixing, and is employed in the present study to test the performance of AHI-S when extinction and re-ignition are involved. The governing equations of unsteady PSRs can be expressed as $[37,38]$ 


$$
\begin{gathered}
\frac{d y_{k}}{d t}=\frac{\omega_{k} W_{k}}{\rho}+\frac{y_{k}^{0}-y_{k}}{\tau_{r e s}}, k=1,2, \ldots N \\
\frac{d T}{d t}=-\frac{\sum_{k=1}^{N} h_{k} \omega_{k} W_{k}}{\rho c_{p}}+\frac{\sum_{k=1}^{N} y_{k}^{0}\left(h_{k}^{0}-h_{k}\right)}{c_{p} \tau_{\text {res }}}
\end{gathered}
$$

where $\tau_{\text {res }}=\rho V / \dot{m}_{\text {in }}$ is a nominal residence time, $V$ is the volume of the reactor, $\dot{m}_{i n}$ is the inlet mass flow rate, and the superscript " 0 " indicates the inlet conditions, which are provided in Table 1 for the different mechanisms tested. Pressure is constant and density is solved by the ideal gas law. The residence time is forced to oscillate sinusoidally in the logarithmic scale as

$$
\log _{10} \tau_{\text {res }}=\log _{10} \tau_{0}+A \cos \left(2 \pi t / \tau_{p}\right)
$$

where $\tau_{0}=10^{-4} \mathrm{~s}, A=2$, and $\tau_{p}$ is the period of oscillation. $\tau_{p}=3 \times 10^{-3} \mathrm{~s}$ is used for the 32-species skeletal mechanism for ethylene, USC-Mech II with methane as fuel, and the 2878species biodiesel mechanism, while $\tau_{p}=10^{-3} \mathrm{~s}$ is used for all the other mechanisms. The selection of the oscillation period is to ensure that both ignition and extinction occur in the simulations.

For unsteady PSRs, the solution procedure of the AHI-S method is similar to that in autoignition calculations. The system of differential algebraic equations (DAE) includes Eqs. (9, 11, 15-16), and the dependent variables are $\boldsymbol{\Phi}=\left[y_{k}, T, \rho, C\right]^{\mathrm{T}}$. Fast variables are treated implicitly and slow variables, including slow species, temperature, density and the total species mole concentration, are solved explicitly.

For comparison, the unsteady PSR system described by Eqs. (15-16) and the ideal gas law with dependent variables $\boldsymbol{\Phi}=\left[y_{k}, T\right]^{\mathrm{T}}$ are also solved using the Strang splitting scheme, in which the following equations are solved sequentially for each time step from $t^{n}$ to $t^{n+1}$ :

$$
\frac{d \boldsymbol{\Phi}^{(1)}}{d t}=\boldsymbol{S}\left(\boldsymbol{\Phi}^{(1)}\right), \quad \boldsymbol{\Phi}^{(1)}(0)=\boldsymbol{\Phi}^{n} \text { on }[0, \Delta t / 2]
$$




$$
\begin{array}{lll}
\frac{d \boldsymbol{\Phi}^{(2)}}{d t}=\boldsymbol{M}\left(\boldsymbol{\Phi}^{(2)}\right), & \boldsymbol{\Phi}^{(2)}(0)=\boldsymbol{\Phi}^{(1)}(\Delta t / 2) \text { on }[0, \Delta t] \\
\frac{d \boldsymbol{\Phi}^{(3)}}{d t}=\boldsymbol{S}\left(\boldsymbol{\Phi}^{(3)}\right), & \boldsymbol{\Phi}^{(3)}(0)=\boldsymbol{\Phi}^{(2)}(\Delta t) \text { on }[0, \Delta t / 2]
\end{array}
$$

where the chemistry and transport terms, $\boldsymbol{S}$ and $\boldsymbol{M}$, are given in Eqs. (15-16). Eq. (18a) is a reaction substep taking the composition $\boldsymbol{\Phi}^{n}$ at time $t^{n}$ as the initial condition. Eq. (18b) is a transport substep with the initial condition taken from the solution of Eqs. (18a). Eq. (18c) is the second reaction substep with the initial condition taken from the solution of Eqs. (18b). At the end of a splitting step, $\boldsymbol{\Phi}^{(3)}(\Delta t / 2)$ is taken as the composition $\boldsymbol{\Phi}^{n+1}$ at time $t^{n+1}$. The reaction substeps in Eqs. (18a) and (18c) are solved by stiff ODE solvers such as VODE and LSODES, while the transport substep in Eq. (18b) is solved by the explicit second-order Runge-Kutta method.
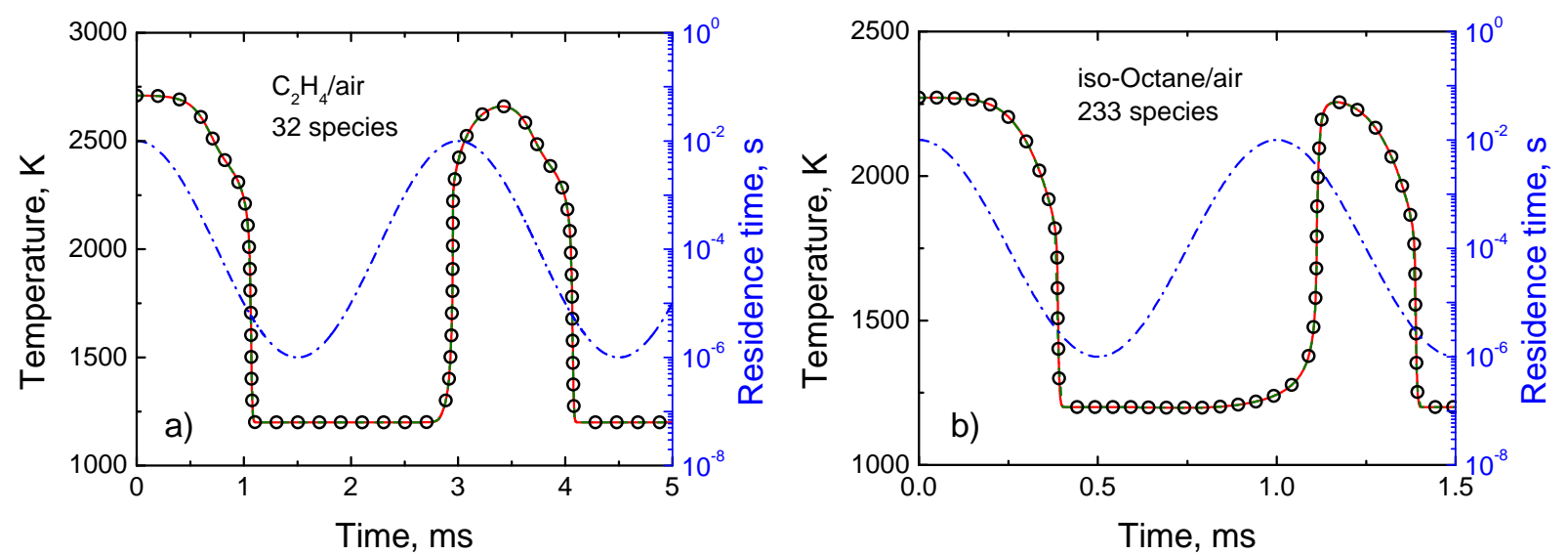

Figure 11. Temperature profiles of unsteady PSRs for a) the 32-species skeletal mechanism for ethylene and b) the 233-species skeletal mechanism for iso-octane, calculated using AHI-S (symbols), VODE (solid lines) and the Strang splitting scheme (dashed lines). A fixed integration time step of $10^{-7} \mathrm{~s}$ is used in AHI-S and the Strang splitting scheme, and the built-in adaptive time stepping algorithm is used in VODE. The dash dot lines represent the fluctuating residence time as function of time. 
Figure 11 shows the temperature profiles in unsteady PSRs for ethylene/air (Fig. 11a) and iso-octane/air (Fig. 11b), calculated with AHI-S, the fully implicit VODE solver, and the Strang splitting scheme with chemistry substeps solved by VODE, which is widely adopted in 2- and 3dimensional flame simulations. The initial conditions are taken from the steady-state solutions of PSR with $\tau_{\text {res }}=10^{-2} \mathrm{~s}$. It is seen that the solutions obtained with AHI-S and the Strang splitting scheme agree well with the solutions obtained with the fully implicit solver for the tested cases, while further discussion on the accuracy of the different solvers can be found in Ref. [16]. The following study will be focused on the comparison of the numerical efficiencies of the different solvers in unsteady PSRs.

Figures $12 \mathrm{a}$ and $12 \mathrm{~b}$ show the computational cost and speed-up factors, respectively, of AHI$\mathrm{S}$ as function of mechanism size, compared with the original AHI, and the Strang splitting scheme. The chemistry substeps in the splitting scheme is solved by VODE using dense matrix operations with numerical and analytic Jacobian, respectively or LSODES with analytic Jacobian. It is seen that the use of analytic Jacobian can improve the computational efficiency for small to moderate sized mechanisms, and AHI can further reduce the computational cost by more than a factor of ten. LSODES has similar performance with VODE for small mechanisms while it is significantly faster than VODE and the original AHI for large mechanisms. AHI-S with sparse matrix techniques results in an overall linear scaling in computational cost versus mechanism size for unsteady PSRs, similar to that shown in Fig. 6 for auto-ignition. In contrast, an overall scaling of $O\left(N^{3}\right)$ is observed for both AHI and the Strang splitting scheme for large mechanisms. The speed-up factor achieved by AHI-S is approximately 50 for the 9-species hydrogen mechanism and $3 \times 10^{4}$ for the 2878-species biodiesel mechanism, compared with the 
Strang splitting scheme using VODE with numerical Jacobian, and is 10 100 for mechanisms with different number of species, compared with the Strang splitting scheme using LSODES with analytic Jacobian. Such time savings are significantly higher than those observed for AHI-S versus VODE or LSODES in auto-ignition shown in Fig. 7. As an explanation, in addition to the reduced size of the implicit core and the incorporation of sparse matrix techniques, the speedup in unsteady PSRs is also attributed to the elimination of the many, say about 10 100, internal time steps needed by the Strang splitting to relax the fast modes artificially re-activated by adding the integrated transport effect at a discrete point during each splitting step, as discussed in Ref. [16].
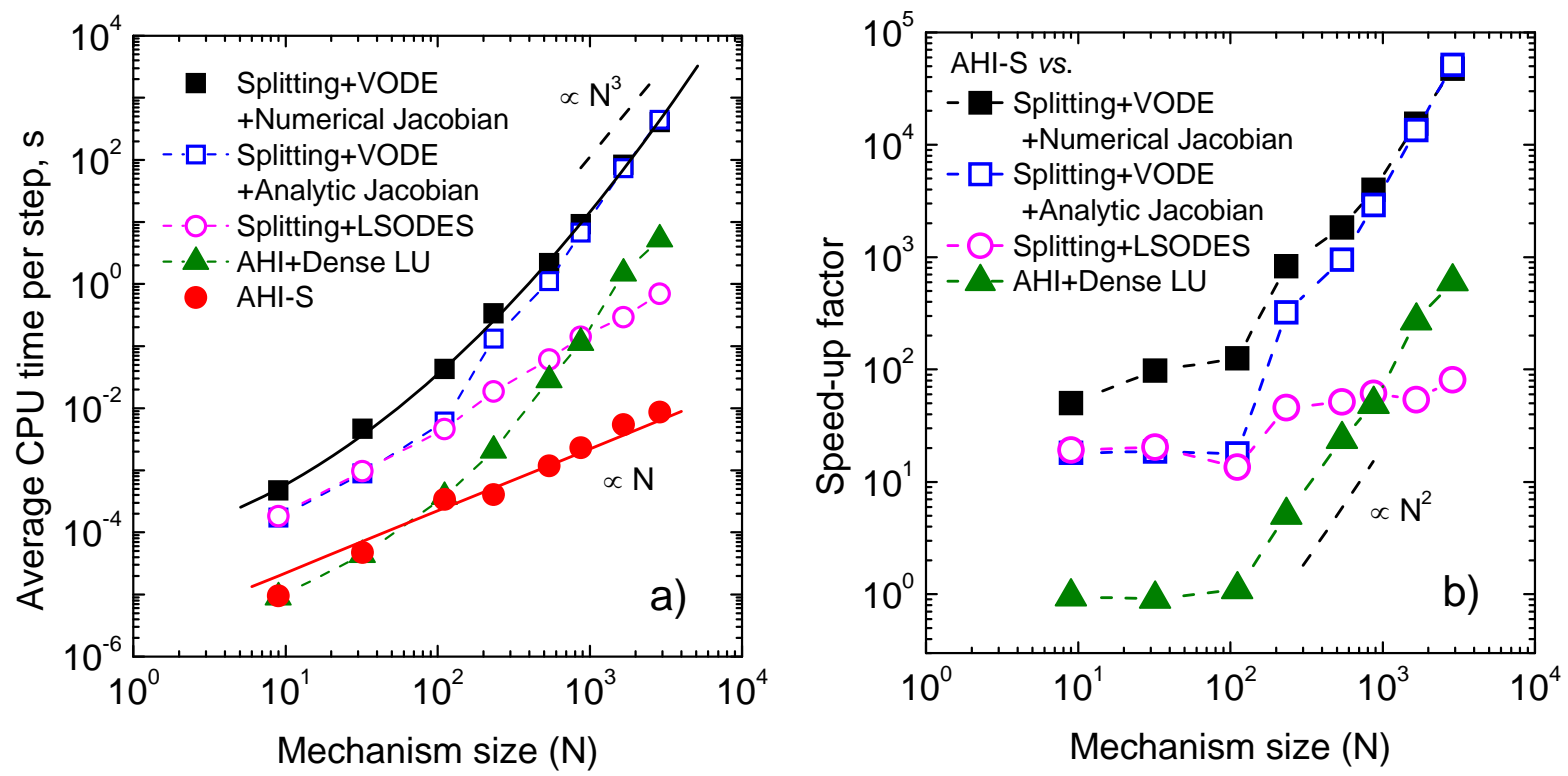

Figure 12. a) Average per-step computational cost for AHI-S, AHI and Strang splitting schemes, and b) speedup factors for AHI-S compared with other solvers, as function of mechanism size for unsteady PSRs.

\subsection{Performance of AHI-S in a 1-D laminar premixed flame}


The effects of convection and diffusion on the numerical performance of AHI-S are further investigated using a 1-D planar unsteady free-propagating premixed flame, for which the governing equations are expressed as

$$
\begin{gathered}
\frac{\partial \rho}{\partial t}+\frac{\partial(\rho u)}{\partial x}=0 \\
\frac{\partial y_{k}}{\partial t}+u \frac{\partial y_{k}}{\partial x}=\frac{1}{\rho} \frac{\partial}{\partial x}\left(\rho D_{k} \frac{\partial y_{k}}{\partial x}\right)+\frac{\dot{\omega}_{k} W_{k}}{\rho}, k=1,2, \ldots N \\
\frac{\partial T}{\partial t}+u \frac{\partial T}{\partial x}=\frac{1}{\rho c_{p}} \frac{\partial}{\partial x}\left(\lambda \frac{\partial T}{\partial x}\right)-\frac{1}{\rho c_{p}} \sum_{k=1}^{N} \dot{\omega}_{k} h_{k} W_{k}
\end{gathered}
$$

where radiative heat transfer, Soret and Dufour effects are ignored, $u$ is velocity, $\lambda$ is heat conductivity, and $D_{k}$ is mixture-averaged diffusivity of the $k^{\text {th }}$ species.

The simulation is performed using the mixture-averaged molecular diffusion model and the detailed USC-Mech II for methane/air with equivalence ratio of 0.6 at pressure of 5 atm and fresh mixture temperature of $300 \mathrm{~K}$. The flame is initialized with the 1-D steady state solution obtained from the PREMIX code [39] in CHEMKIN-II. The computational domain is $[0,1 \mathrm{~cm}]$ with the flame front located at $x=0.2 \mathrm{~cm}$ at $t=0$ and propagating to the $+x$ direction. Symmetric boundary condition is used at the hot boundary $(x=0)$, and wall condition with fixed temperature and species concentrations is specified at the cold boundary $(x=1 \mathrm{~cm})$, as specified in Ref. [11]. Convection and diffusion terms are discretized using the second-order upwind scheme and central difference method, respectively. The convection and diffusion terms are treated as slow source terms in AHI-S in addition to the slow chemistry. Equations $(9,11,19-$ 21) are solved with dependent variables being $\boldsymbol{\Phi}=\left[y_{k}, T, \rho, u, C\right]^{\mathrm{T}}$ at all grid points, where temperature, density, velocity, and the total species mole concentration are treated as slow variables in addition to the slow species. Similar to the procedures used for auto-ignition, in each 
time integration step, the fast variables are first solved implicitly, and the slow variables are then solved explicitly. Specifically, density and the total species mole concentration are updated using Eq. (9) and Eq. (11). Velocity is updated through the continuity equation. That is, velocity in Eq. (19) is implicitly formulated as

$$
\frac{-\rho_{i+2}^{n+1} u_{i+2}^{n+1}+4 \rho_{i+1}^{n+1} u_{i+1}^{n+1}-3 \rho_{i}^{n+1} u_{i}^{n+1}}{2 \Delta x}=-\frac{\rho_{i}^{n+1}-\rho_{i}^{n}}{\Delta t}
$$

where the superscript $n$ indicates the $n$th time step and subscript $i$ indicates the $i$ th grid point, and $\Delta x$ and $\Delta t$ are spatial and temporal resolutions, respectively. This procedure has been demonstrated in Ref. [11] to accurately solve velocity field in the absence of the momentum equation for 1-D low-Mach number flows. Note that for 2- or 3-D flows, the momentum equations are still required to solve for the velocity field. In the Strang splitting scheme, similar procedure as that shown in Eqs. (18a-c) is used except that the transport substep is solved by a second-order Crank-Nicholson method [11].

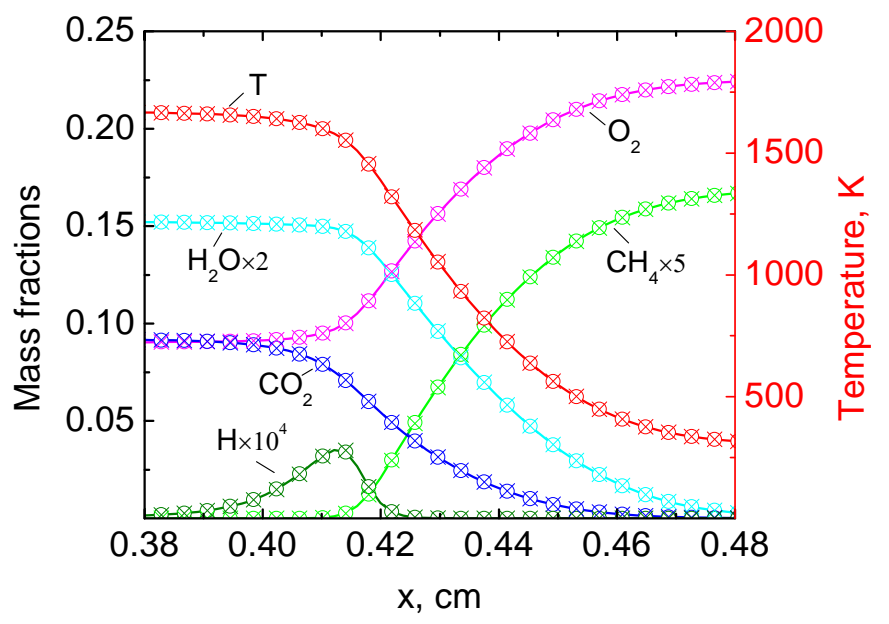

Figure 13. Profiles of temperature and mass fractions of selected species at $t=0.05 \mathrm{~s}$ in a $1-\mathrm{D}$ premixed methane/air flame simulated using USC-Mech II. Circles: $\Delta t=2.5 \times 10^{-8} \mathrm{~s}$. Lines: $\Delta t=10^{-7} \mathrm{~s}$. Crosses: $\Delta t=4 \times 10^{-7} \mathrm{~s}$. 
Figure 13 shows the numerical solutions of the 1-D premixed flame calculated with AHI-S at the $t=0.05 \mathrm{~s}$ using three different integration step sizes, $\Delta t=2.5 \times 10^{-8} \mathrm{~s}, 10^{-7} \mathrm{~s}$ and $4 \times$ $10^{-7} \mathrm{~s}$, respectively. It is seen that the solutions with different temporal resolutions are mostly identical, and thus temporal convergence has been achieved at such fine temporal resolution. Note that the selection of spatial resolution is determined by flame thickness as defined in Ref. [13]:

$$
\delta_{L}=\frac{T_{b}-T_{u}}{\max (|\nabla T|)}
$$

where $T_{b}$ is the flame temperature and $T_{u}$ is the temperature of the fresh mixture. The flame thickness is approximately $386 \mu \mathrm{m}$ based on the temperature profile in Fig. 13 and Eq. (23), and a grid size of $19.5 \mu \mathrm{m}$ is selected such that there are at least 20 grid points across the flame front.

Figure 14 shows the numbers of fast species and fast reactions as function of the spatial coordinate for $\Delta t=10^{-7} \mathrm{~s}$. Since temperature and radical concentrations are both high in the post-flame zone, the numbers of fast species and fast reactions tend to be larger on the product side compared with the reactant side, and rapid changes can be observed across the flame front. This observation is overall consistent with that for auto-ignition as shown in Fig. 9.

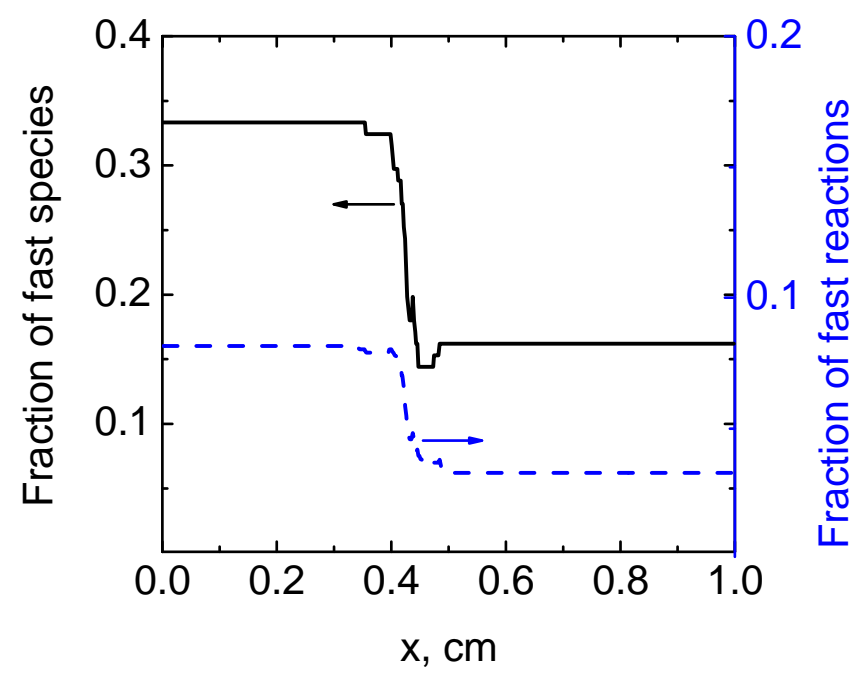


Figure 14. Fractions of fast species and fast reactions as function of the spatial coordinate for the 1-D premixed flame at $t=0.05 \mathrm{~s}$, calculated using USC-Mech II and time step size $\Delta t=1 \times 10^{-7} \mathrm{~s}$.

Figure 15 shows the total computational cost of AHI-S for the entire simulation for $t=[0,0.05 \mathrm{~s}]$, as function of the time step size, compared with that of the Strang splitting scheme with chemistry substeps solved by VODE using numerical Jacobian and dense matrix operations. Speedup by about two orders of magnitude is achieved using AHI-S compared with the Strang splitting scheme, while the speed-up factor is slightly sensitive to the integration step size because the size of fast chemistry in AHI-S increases with time step size. It is noted again that the reason for the significant time saving is threefold: First, the AHI-S scheme solves a smaller implicit core of fast chemistry. Second, the AHI-S scheme eliminates the need to use many internal time steps to relax the artificially activated fast chemical modes needed in the splitting schemes, similar to the unsteady PSR cases. Third, the use of analytic Jacobian combined with sparse matrix techniques can further speed up the simulation for mechanisms of arbitrary sizes.

It is noted that the largest time step size that can be taken in AHI-S in the present study is limited by the fastest transport process (e.g., molecular diffusion) for 1-D premixed flames because the transport processes are treated explicitly, while larger splitting time steps, say $10^{-6} \sim 10^{-5} \mathrm{~s}$, can be used in the Strang splitting scheme with both convection and diffusion solved implicitly [11]. However, for a smaller step size, say $10^{-8} \sim 10^{-6} \mathrm{~s}$, AHI-S can be significantly faster than the Strang splitting scheme.

The above results therefore demonstrated that AHI-S can achieve high numerical efficiency compared with the previous stiff chemistry solvers for both homogeneous systems, such as auto- 
ignition and PSR, and diffusive systems, such as 1-D premixed flames. For 2- and 3-D flames, similar performance of AHI-S is expected if the transport processes are treated explicitly and computational cost of the evaluation of transport source terms is insignificant compared with that of the chemical source term.

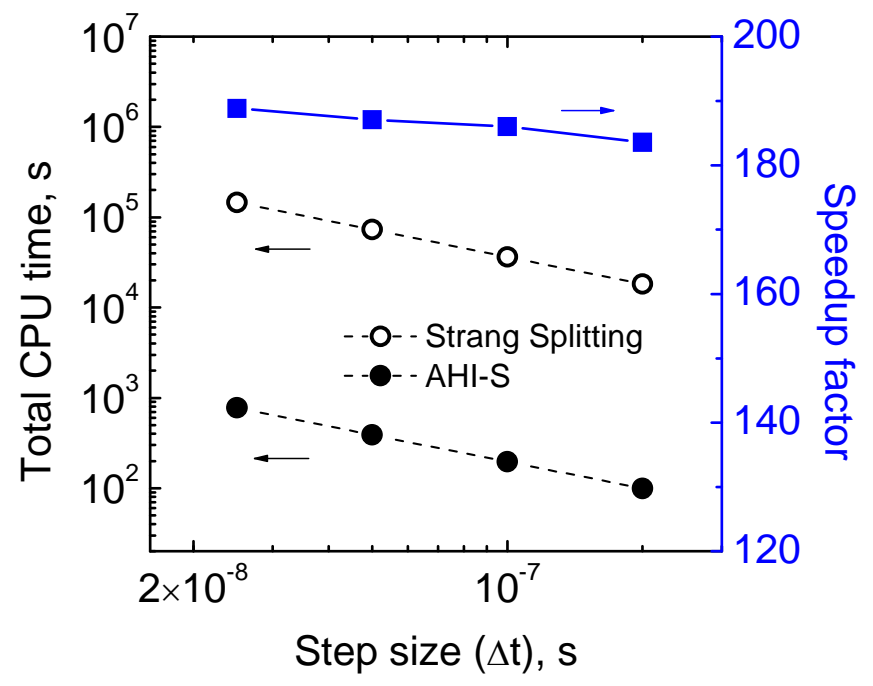

Figure 15. Computational cost (left axis) for the entire simulation of the 1-D premixed flame for $t=[0,0.05 \mathrm{~s}]$ using AHI-S (closed circles) and the Strang splitting scheme (open circles), and the corresponding speedup factors (squares, right axis) using AHI-S compared with the Strang splitting scheme, as function of the time step size, calculated using USC-Mech II for methane/air.

\section{Concluding Remarks}

An AHI-S solver is developed based on a previously developed AHI scheme and sparse matrix techniques. The new solver is tested in various combustion systems, including autoignition, unsteady PSR and 1-D premixed flame propagation using mechanisms of different fuels ranging from 9 to 2878 species. Compared with the original AHI method, AHI-S further reduces 
the nontrivial entries in the Jacobian of the fast chemistry subcomponent to obtain sparser Jacobian of the fast chemistry and to achieve higher computational efficiency.

Tests with constant-pressure auto-ignition show that the per-step computational cost of AHI-S is linearly proportional to mechanism size and is comparable to that of the fully explicit solvers, or a few evaluations of reaction rates using the CHEMKIN-II subroutine, while much implicit-like larger time steps can be adopted by AHI-S similar to other stiff ODE solvers. Compared with the fully implicit LSODES solver, a speed-up factor of approximately 3 is achieved by AHI-S, Compared with the fully implicit VODE solver, speed-up factors ranging from approximately 10 , for the 9 -species hydrogen mechanism, to approximately 3000 , for the 2878-species biodiesel mechanism, are achieved by AHI-S. The time saving is primarily attributed to the reduced size of the implicit core for small to moderate sized mechanisms, say with less than about 100 species, and to the sparse matrix techniques for larger mechanisms.

Further tests with unsteady PSRs and an 1-D unsteady freely propagating premixed flame show that, when non-chemical source terms are present, AHI-S is significantly more efficient than the Strang splitting scheme, which requires many internal steps in the chemistry substep to relax the fast chemical modes re-activated by adding the integrated transport effect in the beginning of the chemistry substep. For unsteady PSRs, speed-up factors ranging from 50 to 28,500 are achieved using AHI-S compared with the Strang splitting scheme with chemistry substeps solved by the fully implicit VODE solver, and speed-up factors of 10 100 are also achieved compared with the Strang splitting scheme using LSODES and analytic Jacobian with different mechanisms. Speed-up factors of approximately 200 are achieved using AHI-S for the 1-D premixed flame of methane/air compared with the Strang splitting scheme using VODE. 
AHI-S can achieve overall explicit-like performance for both small to large mechanisms in different combustion systems, and thus provides a highly efficient approach for time integration of reacting flows involving stiff chemistry.

\section{Acknowledgements}

This work is supported by the Air Force Office of Scientific Research under Grant FA9550-15-10496. The work by Z. Ren is supported by National Natural Science Foundation of China under Grant 51476087 and 91441202 .

\section{References}

[1] T. Lu, C.K. Law, Toward accommodating realistic fuel chemistry in large-scale computations, Prog. Energy Combust. Sci. 35 (2009) 192-215.

[2] T. Lu, C.K. Law, A directed relation graph method for mechanism reduction, Proc. Combust. Inst. $30(2005) 1333-1341$.

[3] P. Pepiot-Desjardins, H. Pitsch, An efficient error-propagation-based reduction method for large chemical kinetic mechanisms, Combust. Flame 154 (2008) 67-81.

[4] W. Sun, Z. Chen, X. Gou, Y. Ju, A path flux analysis method for the reduction of detailed chemical kinetic mechanisms, Combust. Flame 157 (2010) 1298-1307.

[5] R. Sankaran, E.R. Hawkes, J.H. Chen, T. Lu, C.K. Law, Structure of a spatially developing turbulent lean methane-air Bunsen flame, Proc. Combust. Inst. 31 (2007) 1291-1298.

[6] X. Zheng, T. Lu, C. Law, Experimental counterflow ignition temperatures and reaction mechanisms of 1, 3-butadiene, Proc. Combust. Inst. 31 (2007) 367-375. 
[7] K.E. Niemeyer, C.-J. Sung, M.P. Raju, Skeletal mechanism generation for surrogate fuels using directed relation graph with error propagation and sensitivity analysis, Combust. Flame 157 (2010) 1760-1770.

[8] P.N. Brown, G.D. Byrne, A.C. Hindmarsh, VODE: A variable-coefficient ODE solver, SIAM J. Sci. Stat. Comp. 10 (1989) 1038-1051.

[9] M. Caracotsios, W.E. Stewart, Sensitivity analysis of initial value problems with mixed ODEs and algebraic equations, Comput. Chem. Eng. 9 (1985) 359-365.

[10] T. Lu, C.K. Law, C.S. Yoo, J.H. Chen, Dynamic stiffness removal for direct numerical simulations, Combust. Flame 156 (2009) 1542-1551.

[11] Z. Ren, C. Xu, T. Lu, M.A. Singer, Dynamic adaptive chemistry with operator splitting schemes for reactive flow simulations, J. Comput. Phys. 263 (2014) 19-36.

[12] M.A. Singer, S.B. Pope, Exploiting ISAT to solve the reaction-diffusion equation, Combust. Theor. Model. 8 (2004) 361-383.

[13] M.A. Singer, S.B. Pope, H.N. Najm, Operator-splitting with ISAT to model reacting flow with detailed chemistry, Combust. Theor. Model. 10 (2006) 199-217.

[14] O.M. Knio, H.N. Najm, P.S. Wyckoff, A semi-implicit numerical scheme for reacting flow: II. stiff, operator-split formulation, J. Comput. Phys. 154 (1999) 428-467.

[15] Z. Ren, S.B. Pope, Second-order splitting schemes for a class of reactive systems, J. Comput. Phys. 227 (2008) 8165-8176.

[16] Y. Gao, Y. Liu, Z. Ren, T. Lu, A dynamic adaptive method for hybrid integration of stiff chemistry, Combust. Flame 162 (2015) 287-295. 
[17] F. Perini, E. Galligani, R.D. Reitz, An analytical Jacobian approach to sparse reaction kinetics for computationally efficient combustion modeling with large reaction mechanisms, Energy Fuels 26 (2012) 4804-4822.

[18] F. Perini, E. Galligani, R.D. Reitz, A study of direct and Krylov iterative sparse solver techniques to approach linear scaling of the integration of chemical kinetics with detailed combustion mechanisms, Combust. Flame 161 (2014) 1180-1195.

[19] D.A. Schwer, J.E. Tolsma, W.H. Green, P.I. Barton, On upgrading the numerics in combustion chemistry codes, Combust. Flame 128 (2002) 270-291.

[20] V. Damian, A. Sandu, M. Damian, F. Potra, G.R. Carmichael, The kinetic preprocessor KPP-a software environment for solving chemical kinetics, Comput. Chem. Eng. 26 (2002) $1567-1579$.

[21] M.J. McNenly, R.A. Whitesides, D.L. Flowers, Faster solvers for large kinetic mechanisms using adaptive preconditioners, Proc. Combust. Inst. 35 (2015) 581-587.

[22] S. Verma, Y. Xuan, G. Blanquart, An improved bounded semi-Lagrangian scheme for the turbulent transport of passive scalars, J. Comput. Phys. 272 (2014) 1-22.

[23] S.H. Lam, Model reductions with special CSP data, Combust. Flame 160 (2013) 2707-2711.

[24] H. Wang, X. You, A.V. Joshi, S.G. Davis, A. Laskin, F. Egolfopoulos, C.K. Law, USC Mech Version II. High-Temperature Combustion Reaction Model of H2/CO/C1-C4 Compounds, http://ignis.usc.edu/USC_Mech_II.htm, May 2007.

[25] I.S. Duff, J.K. Reid, MA48, a Fortran code for direct solution of sparse unsymmetric linear systems of equations, Science and Engineering Research Council, Rutherford Appleton Laboratory, 1993. 
[26] J. Li, Z. Zhao, A. Kazakov, F.L. Dryer, An updated comprehensive kinetic model of hydrogen combustion, Int. J. Chem. Kinet. 36 (2004) 566-575.

[27] Z. Luo, C.S. Yoo, E.S. Richardson, J.H. Chen, C.K. Law, T. Lu, Chemical explosive mode analysis for a turbulent lifted ethylene jet flame in highly-heated coflow, Combust. Flame 159 (2012) 265-274.

[28] T. Lu, C.K. Law, Linear time reduction of large kinetic mechanisms with directed relation graph: n-Heptane and iso-octane, Combust. Flame 144 (2006) 24-36.

[29] M. Mehl, W.J. Pitz, C.K. Westbrook, H.J. Curran, Kinetic modeling of gasoline surrogate components and mixtures under engine conditions, Proc. Combust. Inst. 33 (2011) 193-200.

[30] M. Mehl, H. Curran, W. Pitz, C. Westbrook, Chemical kinetic modeling of component mixtures relevant to gasoline, in: European Combustion Meeting, 2009.

[31] C. Westbrook, W. Pitz, O. Herbinet, H. Curran, E. Silke, A detailed chemical kinetic reaction mechanism for n-alkane hydrocarbons from n-octane to n-hexadecane, Combust. Flame (2008).

[32] O. Herbinet, W.J. Pitz, C.K. Westbrook, Detailed chemical kinetic oxidation mechanism for a biodiesel surrogate, Combust. Flame 154 (2008) 507-528.

[33] R.J. Kee, F.M. Rupley, J.A. Miller, Chemkin-II: A Fortran chemical kinetics package for the analysis of gas-phase chemical kinetics, Sandia National Labs., Livermore, CA (USA), 1989. [34] A.C. Hindmarsh, ODEPACK, A Systematized Collection of ODE Solvers, in Scientific Computing, R.S. Stepleman et al. (eds.), North-Holland, Amsterdam, 1983 (vol. 1 of IMACS Transactions on Scientific Computation), pp. 55-64.

[35] CHEMKIN-PRO 15112, Reaction Design: San Diego, CA, 2011. 
[36] P.N. Brown, A.C. Hindmarsh, L.R. Petzold, Using Krylov methods in the solution of largescale differential-algebraic systems, SIAM J. Sci. Comput. 15 (1994) 1467-1488.

[37] P. Glarborg, R. Kee, J. Grcar, J. Miller, PSR: a Fortran program for modeling well-stirred reactors, Sandia National Labs., Livermore, CA (USA); Danmarks Tekniske Hoejskole, Lyngby. Lab. of Heating and Air Conditioning, 1986.

[38] J.Y. Chen, Development of reduced mechanisms for numerical modelling of turbulent combustion, in: Workshop on Numerical Aspects of Reduction in Chemical Kinetics, 1997.

[39] R.J. Kee, J.F. Grcar, M. Smooke, J. Miller, E. Meeks, PREMIX: a Fortran program for modeling steady laminar one-dimensional premixed flames, Sandia National Laboratories Report (1985). 